\title{
Correcting the Periodic Optical Distortion for Particle-Tracking Velocimetry in Corrugated-Plate Heat Exchangers
}

\author{
Jaka Pribošek1,* - Miha Bobič² - Iztok Golobič 1 - Janez Diaci1 ${ }^{1}$ \\ ${ }^{1}$ University of Ljubljana, Faculty of Mechanical Engineering, Slovenia \\ 2 Danfoss Trata, Slovenia
}

\begin{abstract}
To improve current methods for the experimental validation of numerical simulations in corrugated-plate heat exchangers (CPHEs), a full-field quantitative velocimetry of fluid flow is required. This paper investigates the application of particle-tracking velocimetry (PTV) to modified CPHEs. For this, an experimental CPHE unit with a transparent, corrugated upper plate was built. We show that by viewing through a corrugated, refractive interface, a complex periodic optical distortion is introduced, that affects and corrupts the estimated particle trajectories. As this problem cannot be addressed using existing calibration techniques, we propose a novel calibration algorithm for periodic optical distortion. The algorithm relies on the automatic detection of nonlinear distortion using a checkerboard target place within the CPHE unit. The calibration is first made on a coarse grid and subsequently refined by a set of low-order periodic basis functions in order to seize the periodic nature of the deformation field. The proposed algorithms have been applied to a test case with known, uniform particle velocities in order to demonstrate the performance. When applied to a real case, a reduction in the systematic positional error by $35 \%$ was demonstrated.

Keywords: optical distortion, corrugated-plate heat exchanger, particle-tracking velocimetry

Highlights

- We modified corrugated-plate heat-exchanger units to allow fluid-flow visualization and applied a particle-tracking velocimetry to measure the flow.

- We addressed the problem of periodic optical distortion due to the view through corrugated refractive interfaces.

- A novel calibration method allowing for periodic optical distortion has been introduced.

- Experimental results using the proposed algorithms show a reduction in the systematic error by $35 \%$.
\end{abstract}

\section{INTRODUCTION}

Plate heat exchangers (PHEs) have recently been gaining more attention owing to their high overall heat-transfer coefficient, compactness and implementation flexibility. As such, they are finally replacing the traditional tubular designs in various process applications [1]. Recent improvements to heat-transfer efficiency, a higher heat-transfer-areato-volume ratio and a lower pressure drop have been achieved by the micro-corrugation of PHE surfaces the so-called corrugated PHE (CPHE) [2].

Design optimization of the corrugated surface is crucial for tailoring this technology for various applications. So far, very few studies have addressed the optimization problem. Such optimizations of PHEs were mainly studied using CFD simulations [3]. However, the validation of these CFD simulations has mainly been limited to the overall pressure drop and to inlet/outlet temperature measurements [4]. To further extend the validation possibilities, the pressure distribution has recently been measured in a CPHE unit [5]. IR thermography has been applied to obtain a deeper understanding of the temperature fields and flow patterns inside the PHE [6] and [7]. In terms of a
CFD validation, the existing methods still have a very limited scope.

The lack of experimental systems aimed at an internal flow inspection was first addressed by Focke and Knibbe [8]. In this study one of the plates of a plate heat exchanger was replaced by its transparent acrylic equivalent, enabling the visualization of the flow patterns inside the PHE. This pioneering study was able to draw many important conclusions and influenced many others that utilized similar experimental setups for the purpose of optimizing CFD codes [9] and [10] or experimental studies on fluid-flow phenomena [11] to [13]. Although most of the reported experimental units enable fluid-flow monitoring, no studies on the velocity fields inside PHEs can be found in the literature. One of the main reasons for this is the challenging system calibration due to the complex optical distortion that occurs in such situations.

Up to now, different optical setups, such as telecentric lenses and Scheimpflug camera configurations [14] to [16], have been exploited to minimize the distortion and compensate for the projection error. In this way, the calibration of a PTV system is fairly straightforward and simple image registration or scaling may be needed. This approach, 
however, does not allow for the compensation of the errors that arise from viewing through refractive interfaces. An analytic study of viewing through a refractive freeform optical interface is presented in [17], while others employ ray-tracing techniques. A generalized system-calibration procedure for optical setups used in particle velocimetry was proposed by Soloff et al. [18], followed by various improvements [19] to [22], as well as various self-calibrating schemes [23]. Existing techniques exploit either polynomial or rational models to model the optical distortion, which is generally sufficient to account for the distortion introduced by either the camera optics or the refractive interfaces, such as optical flats or cylindrical pipes. In the case that more complex, arbitrary distortion occurs, the refractive-matching technique is usually employed to avoid the distortion-compensation problem [24] and [25], since no general optical-distortion models accounting for such cases exist. Extending the work of Soloff et al. in this direction has also been suggested as a further desirable improvement to the PIV and PTV methods [26], although no further studies could be found, to the best of our knowledge.

In an experimental CPHE system, the upper plate is transparent and corrugated periodically, which results in periodic, freeform optical distortion. In our study, we provide experimental evidence that the results from the PTV in the CPHE are highly erroneous when left uncalibrated. In such a situation, the corrugation of the CPHE is small, implying that extremely dense calibration patterns are to be used for the calibration in order to provide sufficient sampling. This often has many practical limitations in regions where total internal reflection due to oblique interfaces occurs, which significantly limits the maximum density of the calibration pattern.

This paper provides a novel method for estimating the periodic optical distortion using a coarse grid pattern, subsequently refined using a discrete cosine transform. This allows us to capture the periodic nature of the deformation fields and still keep a coarse calibration pattern. We believe the proposed study is a first step towards a quantitative fluid-flow inspection inside a CPHE, which would be of great help in the validation of the CFD corrugation optimization.

\section{METHODS}

Our experimental corrugated heat-exchanger unit (Fig. 1) is based on the experimental designs reported in [8], [10] and [11], where the fluid flow is inspected at the cold side of the heat exchanger, and the flow is visualized through a transparent plate. A corrugated surface is first fabricated from 0.5 -mm stainless-steel sheet by means of microforging technology. The corrugation of the plate is two-dimensional with an amplitude of $1.5 \mathrm{~mm}$ and a period of $7 \mathrm{~mm}$ in both the $x$ and $y$ directions fabrication of its transparent counterpart, avoiding the need for costly master-tool fabrication. The master, together with the PMMA sheet, is then preheated to $150{ }^{\circ} \mathrm{C}$ in a ventilated oven. The hot PMMA is then sandwiched between the negative master, on the one side, and the additional glass plate, on the other, and then pressed in a manual clamping press with a $35-\mathrm{kN}$ clamping force and left for $25 \mathrm{~min}$ to cool. Next, the corrugated stainless-steel plate is inserted into the CNC-cut aluminum holder with integrated inlet and outlets and glued with an epoxy that has a high level of aluminum content for a better heat transfer. The pressure drop across the heat exchanger can be monitored with a pressure sensor (PS). The sensor data is acquired with an Agilent DS 34970A connected to a personal computer.
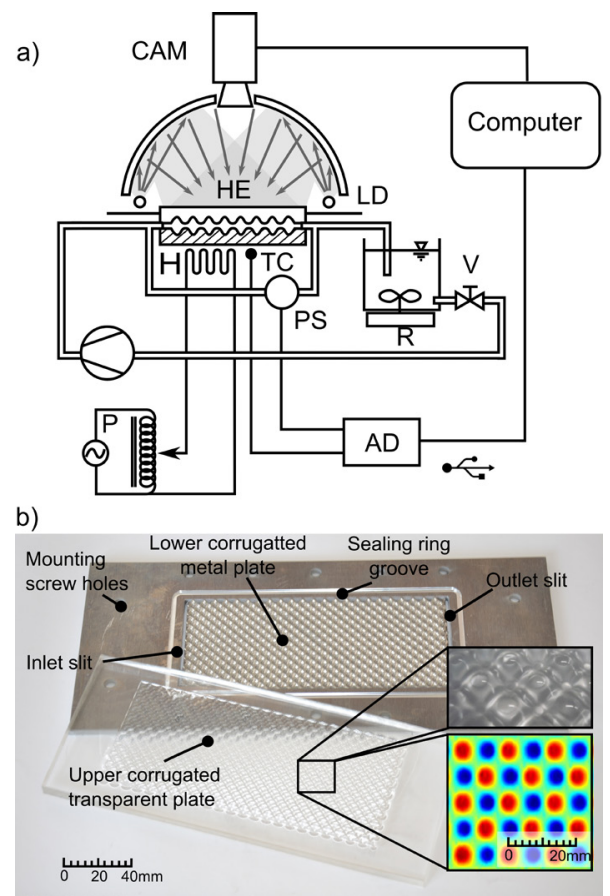

Fig. 1. a) Experimental system for fluid-flow visualization inside the CPHE, and b) fabricated CPHE unit

The fluid flow is monitored using a high-speed camera (Camelopard EVO, Optomotive L.t.d.), featuring 360 FPS streaming for an image size of $2048 \times 1088$ pixels and a pixel size of $5.5 \mu \mathrm{m}$. The camera was equipped with a standard 16-mm C-mount lens. Special attention was given to ensure the proper lighting conditions for the corrugated plate, which 
seems to be a problem that has not received much attention in previous studies [8] and [27]. In order to prevent unwanted shadows and reflections as much as possible, we use homogenous lightning conditions. This was achieved using an improvised lightning tunnel as a variation of the classic Ulbricht Sphere illuminated with eight high-power LED diodes (LD), yielding a high-quality image.

The corrugated, transparent plate in contact with the fluid beneath the plate is a composite optical element that refracts the incident light. If the effects of pressure and temperature are neglected, the light travels in straight rays.

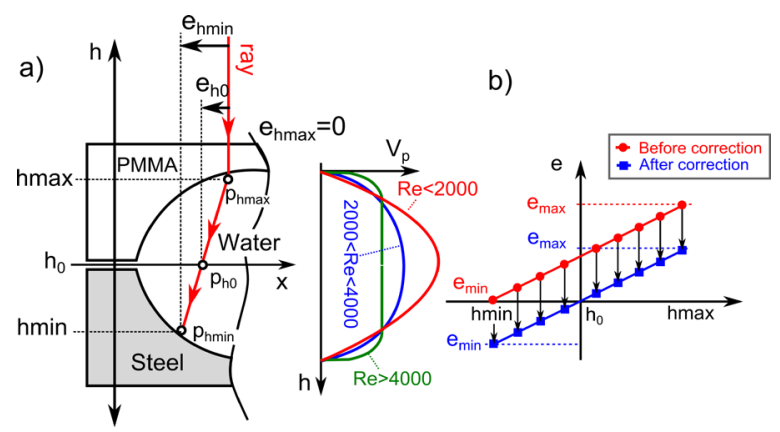

Fig. 2. a) Three particles at different lateral positions along the same optical ray b) Error reduction by distortion compensation

Fig. 2 depicts three particles, $P_{h 0}, P_{h \max }$ and $P_{\text {hmin }}$, along the same optical ray, that all correspond to the same image position on the camera. Regarding their actual position, the errors $E_{h 0}, E_{h \max }$ and $E_{h \min }$ are present in the case of $P_{h \max }$ and $P_{h \min }$, respectively. The error curve is linear, given by $E(h)=k h+e_{h 0}$, and represented by the upper linear curve in Fig. $2 b$. By subtracting the displacement of $P_{h 0}$, the error curve is shifted to zero (see the lower linear curve of Fig. 2b) modelled as $E(h)=k h+e_{h 0}$. What interests us the most, however, is how the calibration affects the total error. This is formulated as an integral of the product of $E(h)$ and the probability $p(h)$ that a particle can be found at one particular height $E_{\text {tot }}=\int_{h \min }^{h \max } E(h) p(h) d h$. Under the assumption of uniformly spread particles in the fluid, the probability $p(h)$ equals the normalized velocity distribution. By inserting the error functions, we estimate the difference between the corrected and uncorrected total errors $E_{\text {tot }}-E_{\text {ctot }}=\int_{h \min }^{h \operatorname{mmax}} e_{h 0} v_{\text {norm }}(h) d h$, which for a positive definite function $v_{\text {norm }}(h)$ is always greater than zero. In highly distributed, turbulent flows, such as in a CPHE with $\operatorname{Re}>4000$, the effects of surface friction are less significant and the velocitydistribution function can be approximated using a uniform function (Fig. 2), for which the corresponding probability function sums to a unit one. As such, by means of a single plane planar calibration we reduced the total error of all the particles, regardless of their height, at least for the term $e_{h 0}(h \max -h \min )$, or greater in the case of less turbulent flows.

\subsection{Optical Distortion Correction}

Given the two different domains, the Image domain $\mathcal{I}$ (observed) and the Cartesian domain $\mathcal{C}$ (hidden state), we refer to the mapping $\mathcal{I} \rightarrow \mathcal{C}$ as direct mapping, and $\mathcal{C} \rightarrow \mathcal{I}$ as the inverse mapping between both domains. We estimate the unknown mappings by observing a fiducial checkerboard pattern through the corrugation plate placed at the middle of the cavity, formed by both plates of the corrugated-plate heat exchangers, yielding exact point-to-point correspondences between both domains.

\subsection{Image Processing}

We have developed a custom algorithm for an automatic mapping estimation based on image processing of the distorted view of a fiducial pattern (Fig. 3). For this a checkerboard pattern was employed, since by considering both white and black regions, a four-times-higher information density is obtained compared to conventional dot patterns. First, an adaptive threshold is applied in order to binarize the image and to eliminate the effects of the illumination gradient that may occur. This was achieved with a $10 \times 10$ window, where the local threshold was set according to the average intensity of that region. After the binarization we apply a morphological erosion to isolate the connected white regions. Two-pass connected component labelling is then applied for their segmentation, allowing us to calculate the centroid point of each blob $i$. The very same procedure is then repeated for the segmentation of the black regions, whereas the morphological erosion was replaced by dilation.

The detected feature points were then meshed using Delaunay triangulation. To each triangle, a circumcircle is calculated. The centroid of the circumcircle is then used to define the nearest neighboring triangle. The two triangles are combined to form a quadrilateral shape, which is then ordered in a grid using the recursive grid-finding algorithm. This grid-finding algorithm starts from an initial, randomly 
chosen $3 \times 3$ neighborhood, which is inserted into an empty matrix $M$. From the remaining neighborhoods in the set, the one with at least one matching node is chosen and inserted into $M$ such that the matching elements overlap. We repeated this procedure in a recursive manner until all the neighborhoods were assigned to $M$. The result of the represented segmentation and meshing is the formation of pointto-point correspondences with a Cartesian uniform mesh required to estimate the optical distortion.

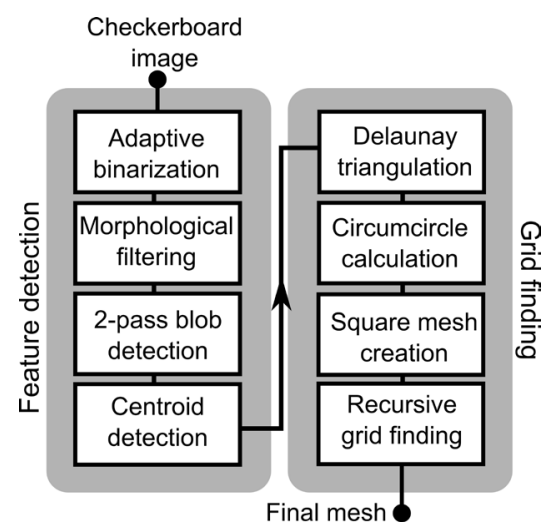

Fig. 3. The complete calibration algorithm

\subsection{Rigid-Body Registration}

By analyzing the point-to-point correspondences between both domains, we attempt to estimate the mapping between them. Fig. 4 shows the major steps of the nonlinear spatial deformation field estimation. Let $p_{i}$ be the observed vertices in the Image domain and $q_{i}$ the corresponding hidden vertices in the Cartesian domain of the same fiducial pattern, for $i=1, \ldots, N$. We may then split the mapping $T$ we are searching for into a rigid-body transformation term $H$ (camera extrinsic parameters) and a nonlinear deformation field $\delta_{i}$ induced by the optical distortion of the corrugated plate $q_{i}=\mathrm{H} p_{i}+\delta_{i}$. Using the homogenous coordinate notation for the given point-to-point correspondences $p_{i}$ and $q_{i}, i=1, \ldots, N$ we have:

$$
\left[\begin{array}{c}
q_{x i} \\
q_{y i} \\
1
\end{array}\right]^{\mathcal{C}}=\left[\begin{array}{ccc}
s_{x} \cos \alpha & -s_{y} \sin \alpha & t_{x} \\
s_{x} \sin \alpha & s_{y} \cos \alpha & t_{y} \\
0 & 0 & 1
\end{array}\right]\left[\begin{array}{c}
p_{x i} \\
p_{y i} \\
1
\end{array}\right]^{\mathcal{I}}+\left[\begin{array}{c}
\delta_{x i} \\
\delta_{y i} \\
0
\end{array}\right]^{\mathcal{C}} .
$$

Assuming an isotropic scaling $s_{x}=s_{y}=s$ we can reformulate the following least-square system, minimizing the $\delta_{i}$ terms, yielding a rigid-body registration that accounts for the camera pose $\left(\alpha, t_{x}, t_{y}\right)$, and the optical magnification $(s)$ :

$$
\left[\begin{array}{cccc}
p_{x 1} & -p_{y 1} & 1 & 0 \\
p_{y 1} & p_{x 1} & 0 & 1 \\
p_{x 2} & -p_{y 2} & 1 & 0 \\
p_{y 2} & p_{x 2} & 0 & 1 \\
\vdots & \vdots & \vdots & \vdots \\
p_{x n} & -p_{y n} & 1 & 0 \\
p_{y n} & p_{x n} & 0 & 1
\end{array}\right]\left[\begin{array}{c}
s \cos (\alpha) \\
s \sin (\alpha) \\
t_{x} \\
t_{y}
\end{array}\right]=\left[\begin{array}{l}
q_{x 1} \\
q_{y 1} \\
q_{x 2} \\
q_{y 2} \\
\vdots \\
q_{x n} \\
q_{y n}
\end{array}\right] .
$$

The above system $A x=y$ is solved for $x$ using the singular value decomposition (SVD) and yields the elements of the rigid-body transformation matrix $H$. This then allows the characterization of the nonlinear terms induced by the optical distortion as the difference between $q$ and $p^{\prime}$ :

$$
\left[\begin{array}{l}
\delta_{x i} \\
\delta_{y i}
\end{array}\right]=\left[\begin{array}{l}
q_{x i} \\
q_{y i}
\end{array}\right]-\left[\begin{array}{c}
p_{x i}{ }^{\prime} \\
p_{y i}{ }^{\prime}
\end{array}\right],
$$

where $p^{\prime}$ denotes the $p$ being transformed to $\mathcal{C}$ :

$$
\left[\begin{array}{c}
\delta_{x i} \\
\delta_{y i} \\
0
\end{array}\right]^{\mathcal{C}}=\left[\begin{array}{c}
q_{x i} \\
q_{y i} \\
1
\end{array}\right]^{\mathcal{C}}-\left[\begin{array}{ccc}
s \cos \alpha & -s \sin \alpha & t_{x} \\
s \sin \alpha & s \cos \alpha & t_{y} \\
0 & 0 & 1
\end{array}\right]\left[\begin{array}{c}
p_{x i} \\
p_{y i} \\
1
\end{array}\right]^{\mathcal{I}},
$$

where $\delta_{x i}$ and $\delta_{y i}$ then represent the required deformation field, evaluated for the region $i$.

\subsection{Parametrization of the Deformation Fields}

In order to ensure good sampling of the deformation field, we strive to keep the fiducial calibration pattern as dense as possible, while still maintaining good performance and robust segmentation. Regardless of all the efforts, the obtained sampling frequency of the optical distortion remains very low. However, since the structures of the corrugated plate have a periodic geometry, the deformation field induced by the optical distortion will be periodic. Since we sample the displacement field with a sampling frequency that is a non-integer multiple of the fundamental frequency of the periodic structures, we may exploit this fact to further improve the estimation of the optical distortion. We do this by modelling a spatial deformation vector field as a linear combination of some periodic basis functions. The basis function used here are a few frequency components $(<300)$ of the two-dimensional direct cosine transform (2D DCT) of the input deformation field. The transform of the variable $\delta_{x}^{\{\mathcal{C}\}}\left(k_{1}, k_{2}\right)$ in the Cartesian domain to $F\left(k_{1}, k_{2}\right)$ in the frequency domain for the 2D DCT is given in [28]: 


$$
\begin{aligned}
& F\left(k_{1}, k_{2}\right)=\alpha\left(k_{1}\right) \alpha\left(k_{2}\right) \sum_{n_{1}=0}^{N_{1}-1 n_{2}=0} \delta_{x}^{\{\mathcal{C}-1}\left(k_{1}, k_{2}\right) \\
& \cos \left(\frac{\pi\left(2 n_{1}+1\right) k_{1}}{2 N_{1}}\right) \cos \left(\frac{\pi\left(2 n_{2}+1\right) k_{2}}{2 N_{2}}\right),
\end{aligned}
$$

for $k_{1}=0,1, \ldots, N_{1}-1$ and $k_{2}=0,1, \ldots, N_{2}-1$ and $\alpha(k)$ defined as:

$$
\alpha(k)=\left\{\begin{array}{lc}
\sqrt{\frac{1}{N}}, & \text { for } k=0 \\
\sqrt{\frac{2}{N}}, & \text { for } k=1,2, \ldots, N-1
\end{array} .\right.
$$

Analogously to Eq. (6), we write the same for the transformation of $\delta_{y}^{\{\mathcal{C}\}}\left(k_{1}, k_{2}\right)$.

Using the 2D DCT, we enforce the periodicity on the estimated "sparse" deformation field by blocking some of the lowest and highest frequency components. Using the filtered $\tilde{F}\left(k_{1}, k_{2}\right)$ we reproduce the filtered deformation field as:

$$
\begin{aligned}
& \widetilde{\delta}_{x}^{\{\mathcal{C}\}}\left(k_{1}, k_{2}\right)=\sum_{k_{1}=0}^{N_{1}-1 \sum_{2}=0} \alpha\left(k_{1}\right) \alpha\left(k_{2}\right) \tilde{F}\left(k_{1}, k_{2}\right) \\
& \cos \left(\frac{\pi\left(2 n_{1}+1\right) k_{1}}{2 N_{1}}\right) \cos \left(\frac{\pi\left(2 n_{2}+1\right) k_{2}}{2 N_{2}}\right),
\end{aligned}
$$

for $n_{1}=0,1, \ldots, N_{1}-1$ and $n_{2}=0,1, \ldots, N_{2}-1$.

In addition to this, knowing the deformation field as an linear combination of some basis functions allows us to upsample the original deformation field (Fig. 5b), while maintaining the smoothness and periodicity constraints. Since the parametrization was made in the Cartesian domain, we apply a backward transform to the image domain, and interpolate it over the entire domain to prepare the look-up table for an efficient implementation.
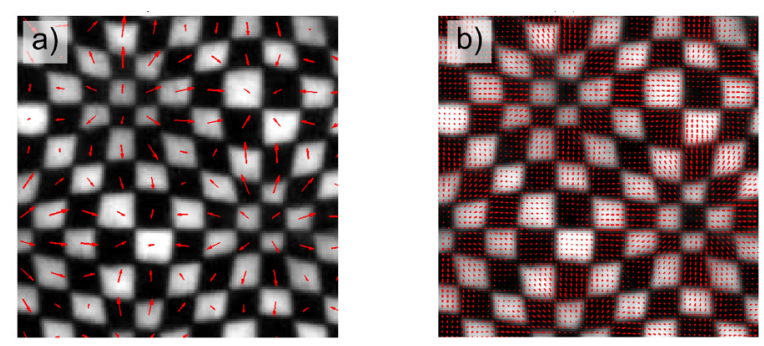

Fig. 5. a) Original and b) upsampled and smoothed deformation field; the magnitude of the deformation field on right was scaled down for the sake of clarity

After the calibration we acquire an image stream of the particles flowing through the CPHE section.
The outcome of the general PTV algorithm is a state vector built from particle positions and velocities in the image domain $\mathcal{I}$ :

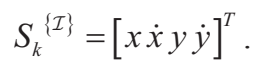

where $k$ runs through all the detected particles. To allow an estimation of the true physical quantities in the Cartesian domain, the trajectories are corrected by the mapping build from the deformation fields $\widetilde{\delta_{x}}$ and $\widetilde{\delta_{y}}$ :

$$
\left[\begin{array}{c}
s_{k}^{\prime} \\
1
\end{array}\right]^{\{C\}}=\left[\begin{array}{ccccc}
s_{x} & 0 & 0 & 0 & \widetilde{\delta}_{x}(x, y) \\
0 & s_{x} & 0 & 0 & \widetilde{\delta_{x}}(\mathrm{x}+\dot{\mathrm{x}} \mathrm{t}, \mathrm{y}+\dot{y} \mathrm{t})-\widetilde{\delta}_{x}(x, y) \\
0 & 0 & s_{y} & 0 & \widetilde{\delta}_{y}(x, y) \\
0 & 0 & 0 & s_{y} & \widetilde{\delta_{y}}(\mathrm{x}+\dot{\mathrm{x}}, \mathrm{y}+\dot{y} \mathrm{t})-\widetilde{\delta}_{y}(x, y) \\
0 & 0 & 0 & 0 & 1
\end{array}\right],
$$

\section{EXPERIMENTAL}

\section{$2.1 \quad$ Optical Distortion Estimation}

We inspect the optical distortion at the central section plane of the CPHE unit, in such a way that the fiducial pattern is pressed against the upper corrugated plate. Fig. 6 shows the periodically distorted view of the calibration pattern. As seen from Fig. 6, the distortion cannot be neglected as it would result in erroneous particle trajectories and velocities. The distortion compensation is thus crucial and is discussed in Section 4.2.

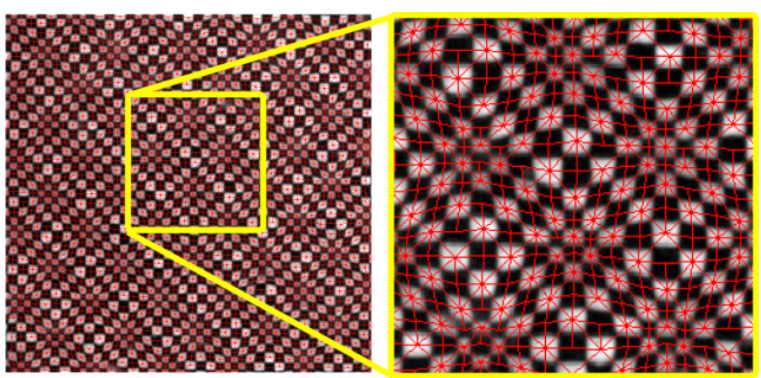

Fig. 6. Periodically distorted view of the fiducial calibration pattern observed through CPHE; the image is overlaid with an automatically detected mesh that corresponds to the Cartesian grid of the fiducial pattern

\subsection{Evaluation of the PTV Correction Algorithm}

We tested the performance of the proposed methods in a test case involving the one-dimensional uniform flow of fiducial particles. First, the system was calibrated as described in Section 4.1 in a central section plane of the heat-exchanger unit. Next, a 
fiducial, randomized polka-dot pattern was placed below the corrugated plate at different heights from the central section, i.e., $0.5 \mathrm{~mm}, 1 \mathrm{~mm}, 1.5 \mathrm{~mm}$ and $2.0 \mathrm{~mm}$.

In this way the effects of the particle flow at various heights were simulated. The pattern is then translated in the $x$ direction in steps of $500 \mu \mathrm{m}$ using a precision micro-translation stage. This means that the simulated velocity-field distribution of the particles is known to be uniform and one dimensional (no $x$-velocity components present). This allows us to evaluate the spatial errors of the particle trajectories before and after the correction. To each trajectory, a line has been fitted, and the deviation from the line has been analyzed. The mean total error in Table 1 is then calculated as the mean of all the absolute deviations from the straight lines.
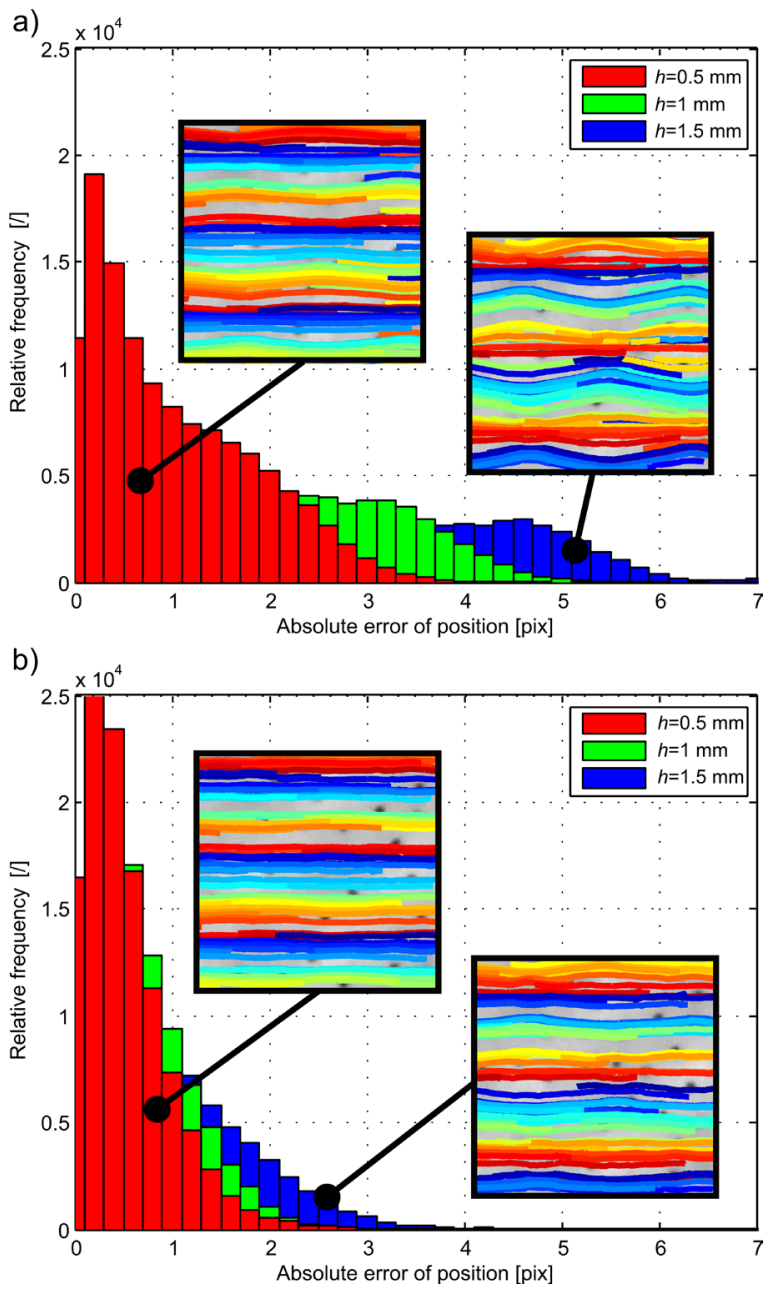

Fig. 7. Absolute error of the position in test-case scenario a) before and b) after distortion correction (see online journal for color version of this figure)

\section{DISCUSION}

Fig. 7 shows the total error evaluated for all the streamlines before the correction. Here, the total error increases with the depth of the particles. Fig. 8 represents the same trajectories after the distortion compensation. The distortion was measured at the central section plane $(h=0)$, so the positional errors are reduced in proportion to the particle height. Table 1 represents the exact percentage of the error elimination that is gained by the distortion correction.

Table 1. Mean total error before and after correction

\begin{tabular}{ccccc}
\hline & $\begin{array}{c}\text { Height } \\
{[\mathrm{mm}]}\end{array}$ & $\begin{array}{c}\text { Mean total } \\
\text { error before } \\
\text { correction [pix] }\end{array}$ & $\begin{array}{c}\text { Mean total } \\
\text { error after } \\
\text { correction [pix] }\end{array}$ & $\begin{array}{c}\text { Percentage } \\
\text { error } \\
\text { eliminated [\%] }\end{array}$ \\
\hline \multirow{3}{*}{ Test case } & 0.5 & 1.0 & 0.51 & 51.0 \\
\cline { 2 - 5 } & 1 & 1.54 & 0.60 & 61.1 \\
\cline { 2 - 5 } & 1.5 & 2.12 & 0.87 & 58.9 \\
\hline Real case & 1 & 5.75 & 3.99 & 30.6 \\
\hline
\end{tabular}

Applying the same principle to the real case, the percentage difference before and after the correction is less significant. This is due to the fact that some

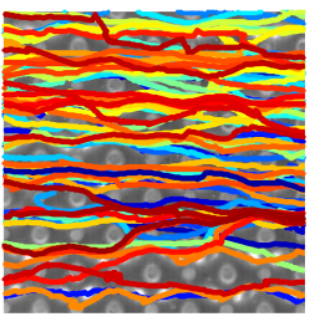

Before correction

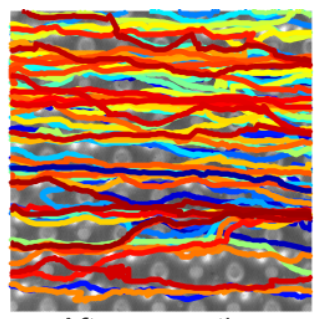

After correction

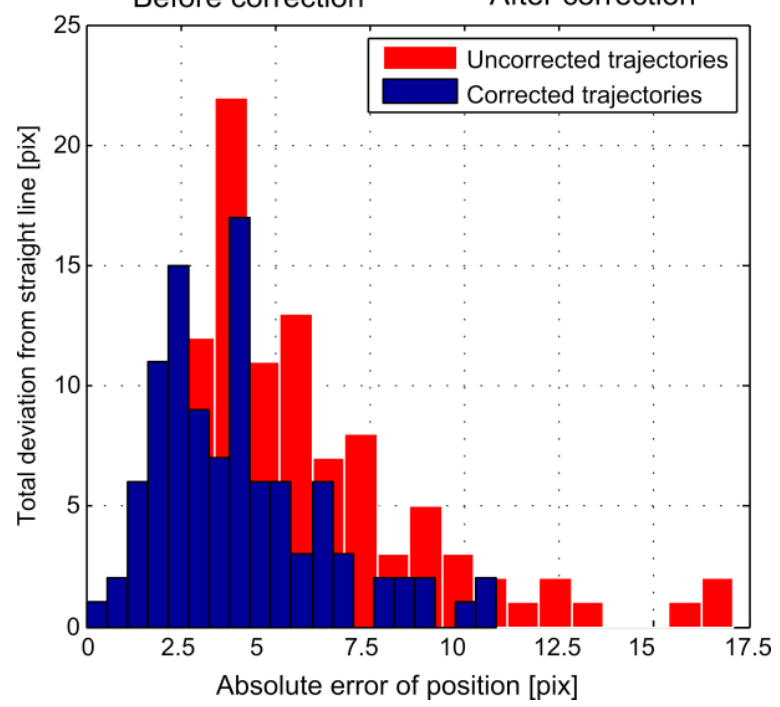

Fig. 8. Absolute error in real case before and after the correction (see online journal for color version of this figure) 
particles flow above the central section plane, and when calibrated, a small positional error is introduced, as clarified in Section 1. Last but not least, it is worth mentioning that by knowing the exact height of each individual particle, obtained using any type of $3 \mathrm{D}$ velocimetry method, the error of the particle trajectory could be completely eliminated. For this, a volumetric calibration would be required, which could be easily performed by applying the same calibration procedures at multiple heights.

\section{CONCLUSION}

In this study we report on the design of an experimental framework to study the fluid-flow phenomena inside a corrugated-plate heat exchanger. We analyzed the error of the conventional particle-tracking velocimetry due to the optical distortion induced by the corrugated optical interface. We developed an automatic calibration algorithm in order to estimate the nonlinear periodic deformation field, used to partially eliminate the positional error of the particletracking velocimetry. The presented experimental framework and methods enable a deeper insight into the flow phenomena inside corrugated PHEs and facilitate experimental studies and the optimization of corrugated-plate heat exchangers. Further work towards 3D velocimetry would open up possibilities to eliminate the error completely.

\section{REFERENCES}

[1] Wang, L., Sundén, B., Manglik, R.M. (Eds.) (2007). Plate Heat Exchangers: Design, Applications and Performance. WIT Press, Southampton.

[2] Ansari, M.S., Mishra, P., Gaikwad, K., Awachat, P.N. (2014). Heat transfer analysis of corrugated plate heat exchanger over coil type heat exchanger: A review. International Journal of Engineering Research and Technology, vol. 3, no. 3, p. 446449.

[3] Aslam Bhutta, M.M., Hayat, N., Bashir, M.H., Khan, A.R., Ahmad, K.N., Khan, S. (2012). CFD applications in various heat exchangers design: A review. Applied Thermal Engineering, vol. 32, p. 1-12, D0l:10.1016/j.applthermaleng.2011.09.001.

[4] Gullapalli, V.S., Sundén, B. (2014). CFD simulation of heat transfer and pressure drop in compact brazed plate heat exchangers. Heat Transfer Engineering, vol. 35, no. 4, p. 358366, DOl:10.1080/01457632.2013.828557.

[5] Ahn, C.H., Choi, J., Son, C., Min, J.K., Park, S. H., Gillespie, D., Go, J.S. (2013). Measurement of pressure distribution inside a cross-corrugated heat exchanger using microchannel pressure tappings. Measurement Science and Technology, vol. 24, no. 3, p. 035306, DOl:10.1088/0957-0233/24/3/035306.

[6] Kabelac, S. (2010). Analysis of local heat transfer in plate heat exchangers for flow pattern optimisation. 14th International
Heat Transfer Conference, vol. 4, p. 375-384, Dol:10.1115/ IHTC14-22387.

[7] Freund, S., Kabelac, S. (2010). Investigation of local heat transfer coefficients in plate heat exchangers with temperature oscillation IR thermography and CFD. International Journal of Heat and Mass Transfer, vol. 53, no. 19-20, p. 3764-3781, D0l:10.1016/j.ijheatmasstransfer.2010.04.027.

[8] Focke, W.W., Knibbe, P.G. (1986). Flow visualization in parallelplate ducts with corrugated walls. Journal of Fluid Mechanics, vol. 165, p. 73-77, DOl:10.1017/S0022112086003002.

[9] Paras, S.V., Kanaris, A.G., Mouza, A.A., Karabelas, A.J. (2002). CFD code application to flow through narrow channels with corrugated walls. 15th International Congress of Chemical and Process Engineering.

[10] Kanaris, A.G., Mouza, K.A., Paras, S.V. (2004). Designing novel compact heat exchangers for improved efficiency using a CFD code. $1^{\text {st }}$ International Conference From Scientific Computing to Computational Engineering.

[11] Vlasogiannis, P., Karagiannis, G., Argyropoulos, P., Bontozoglou, V. (2002). Air-water two-phase flow and heat transfer in a plate heat exchanger. International Journal of Multiphase Flow, vol. 28, no. 5, p. 757-772, Dol:10.1016/ S0301-9322(02)00010-1.

[12] Bellows, K.D. (1997). Flow Visualization of Louvered-Fin Heat Exchangers. University of Illinois, Urbana.

[13] Subbiah, M. (2012). The characteristics of brazed plate heat exchangers with different chevron angles. Heat Exchangers Basics Design Applications, INTECH Open Access Publisher, Rijeka, DOI:10.5772/32888.

[14] Prasad, A.K., Jensen, K. (1995). Scheimpflug stereocamera for particle image velocimetry in liquid flows. Applied Optics, vol. 34, no. 30, p. 7092-7099, Dol:10.1364/a0.34.007092.

[15] Astarita, T. (2012). A Scheimpflug Camera Model for Stereoscopic and Tomographic PIV. 16 th International Symposium on Applications of Laser Techniques to Fluid Mechanics.

[16] Konrath, R. Schröder, W. (2002). Telecentric lenses for imaging in particle image velocimetry: a new stereoscopic approach. Experiments in Fluids, vol. 33, no. 5, p. 703-708, DOl:10.1007/s00348-002-0531-7.

[17] Agarwal, S., Mallick, S.P., Kriegman, D., Belongie, S. (2004). On Refractive Optical Flow. Computer Vision-ECCV, Springer, Berlin, Heidelberg, p. 483-494, D0l:10.1007/978-3-54024671-8_38.

[18] Soloff, S.M., Adrian, R.J., Liu, Z.-C. (1997) Distortion compensation for generalized stereoscopic particle image velocimetry. Measurement Scienceand Technolgy, vol. 8, no. 12, p. 1441-1454.

[19] Willert, C.E. (2006). Assessment of camera models for use in planar velocimetry calibration. Experiments in Fluids, vol. 41, no. 1, p. 135-143, D0I 10.1007/s00348-006-0165-2.

[20] Claus, D., Fitzgibbon, A.W. (2005). A rational function lens distortion model for general cameras. IEEE Computer Society Conference on Computer Vision and Pattern Recognition, vol. 1, p. 213-219, D0l:10.1109/CVPR.2005.43.

[21] Krug, D., Holzner, M., Lüthi, B., Wolf, M., Tsinober, A., Kinzelbach, W. (2014). A combined scanning PTV/LIF technique to simultaneously measure the full velocity gradient 
tensor and the 3D density field. Measurement Science and Technology, vol. 25, no. 6, p. 065301, Dol:10.1088/09570233/25/6/065301.

[22] Schanz, D., Gesemann, S., Schröder, A., Wieneke, B., Novara, M. (2013). Non-uniform optical transfer functions in particle imaging: calibration and application to tomographic reconstruction. Measurement Science and. Technology, vol. 24, no. 2, p. 024009, Dol:10.1088/0957-0233/24/2/024009.

[23] Wieneke, B. (2008). Volume self-calibration for 3D particle image velocimetry. Experiments in Fluids, vol. 45, no. 4, p. 549-556, DOI:10.1007/s00348-008-0521-5.

[24] Budwig, R. (1994). Refractive index matching methods for liquid flow investigations. Experiments in Fluids, vol. 17, no. 5, p. 350-355, DOl:10.1007/BF01874416.

[25] Hassan, Y. A., Dominguez-Ontiveros, E. E. (2008). Flow visualization in a pebble bed reactor experiment using PIV and refractive index matching techniques. Nuclear Engineeringand Design, vol. 238, no. 11, pp. 3080-3085, D0l:10.1016/j. nucengdes.2008.01.027.

[26] Adrian, R. J. (2005). Twenty years of particle image velocimetry. Experiments in Fluids, vol. 39, no. 2, p. 159-169, D0l:10.1007/s00348-005-0991-7.

[27] Pantzali, M.N., Kanaris, A.G., Antoniadis, K.D., Mouza, A.A., Paras, S.V. (2009). Effect of nanofluids on the performance of a miniature plate heat exchanger with modulated surface. International Journal of Heat and Fluid Flow, vol. 30, no. 4, p. 691-699, D0I:10.1016/j.ijheatfluidflow.2009.02.005.

[28] Ashburner, J., Friston, K.J. (1999). Nonlinear spatial normalization using basis functions. Human Brain Mapping, vol. 7, no. 4, p. 254-266, Dol:10.1002/(SICI)10970193(1999)7:4<254::AID-HBM4>3.0.C0;2-G. 